\title{
Complementary and Alternative Treatment of Using BIOCERAMIC related Technology on Mental and Psychiatric Related Disorders
}

\author{
Ting-Kai Leung ${ }^{1,2,3 *}$ \\ ${ }^{1}$ Department of Radiology, Taoyuan General Hospital, Ministry of Health and Welfare, No.1492, Zhongshan Rd., Taoyuan Dist., \\ Taoyuan City 330, Taiwan. \\ ${ }^{2}$ Graduate Institute of Biomedical Materials and Tissue Engineering, College of Biomedical Engineering, Taipei Medical \\ University, Taipei, Taiwan \\ ${ }^{3}$ College of Health Care and Management, Kainan University, 33857 No.1 Kainan Rd. Luzhu, Taoyuan, Taiwan
}

\begin{abstract}
Asian Journal of Complementary and Alternative Medicine. Volume 09 Issue 1
\end{abstract}
Published on: 25/02/2021

*Author for Correspondence: Ting-Kai Leung, Department of Radiology, Taoyuan General Hospital, Ministry of Health and Welfare, No.1492, Zhongshan Rd., Taoyuan Dist., Taoyuan City 330, Taiwan

Cite this article as: Leung TK. Complementary and Alternative Treatment of Using BIOCERAMIC related Technology on Mental and Psychiatric Related Disorders. Asian Journal of Complementary and Alternative Medicine, Vol 9(1), 24-27:2021.

\begin{abstract}
Complementary and alternative medicine treatments are in progress of applications on different mental and psychiatric related problems, include sleep problem, anxiety and depression. Most patients with mental health problems are using both conventional and alternative therapies. As a kind of complementary and alternative medical treatment, devices of photoluminescent of BIOCERAMIC and BIOCERAMIC resonance are based on BIOCERAMIC material, which was begun from in vitro cell study, and then go through different animal and human trials. Herein, a review is to conclude our previous studies on therapeutic effect of BIOCERAMIC related technology on mental and psychiatric related disorders.
\end{abstract}

\section{INTRODUCTION}

In America, there is over one third of population had received complementary and alternative medicine (CAM) treatments, and it is more common among people with mental and psychiatric related problems because of sleep problem, anxiety and depression. Most patients with mental health problems are using both conventional and CAM therapies [13]. An American study was reported that $11 \%$ patients with schizophrenia and $30 \%$ patients of affective disorder had consulted for CAM care for either physical or psychiatric symptoms [4]. It was also reported that Traditional Chinese medical skill of acupuncture was shown its ability of relaxant effect and reduce sleep difficulty in anxious patients, therefore, represent an example of CAM to pharmaceutical therapy for mental health problem [5].

\section{BIOCERAMIC related technology}

BIOCERAMIC technology refers to a material with biological effects, is able to produce non-ionized, weak force fields that cause different biophysical and systemic health benefits with the fundamental effect to weaken hydrogen bonds, thus altering the characteristics of water, as well as those of interstitial fluid and the dynamics of the microcirculation [6]. Since BIOCERAMIC has the characteristics of luminescent effect with photoexcitation within infra-red ray or other light wave spectrum, which has the characteristics of deep optical penetration depth. Thus, device of 'Photoluminescent of BIOCERAMIC material' (PLB) was then developed. PLB uses a visible light source of light-emitting diodes (LEDs), which emit a wavelength of a visible light spectrum between 390 to 750 $\mathrm{nm}$ [7]. Moreover, BIOCERAMIC could also amplify during sound wave pass through the BIOCERAMIC material and then propagate to long distance or deeper penetration of living tissue and then further resonance with physiological rhythms of heart rate brainwaves of human for optimal energy transfer [7]. By selecting specific tempo frequencies of sound wave, device of BIOCERAMIC resonance produces vibration of specific meridian channels described by Traditional Chinese medicine (TCM), which brings to clinical benefits $[7,8]$. Our study was then begun from in vitro cell study of material science, and then go through different animal and human trials[6], There were under permissions with a series of IRB approvals, include Taipei Medical University \& Hospital, Taipei Hospital and Taoyuan General Hospital [6-14]. In this review article, we present our experience base on clinical 
experimental results to explain and describe clinical efficacy BIOCERAMIC related technology that apply to mental and psychiatric related disorders[9-14].

\section{In vivo animal study of BIOCERAMIC material to alleviate stress conditions}

Like human, the emotional stress of the rat induced by auditory and visual stimulation, is obviously associated with rapid heart rate, tachycardia and myocardial ischemia. This condition could be monitored by in-vivo cardiovascular hemodynamic parameters in rats, include real time data of systolic blood pressure, mean blood pressure, diastolic blood pressure and heart rate under stress by indirect cardiovascular hemodynamic parameters that measured in conscious rats using tail plethysmography with tail-cuff method by applying a non-preheated and noninvasive blood pressure manometer. Cardiovascular hemodynamic parameters were recorded in rats without (control group) or with (BIOCERAMIC group) BIOCERAMIC silicon rubber plates during two 11-min recording periods. BIOCERAMIC irradiation caused a significant decrease in the average heart rate of the rats subjected to auditory and visual stress. Moreover, the stress induced high systolic blood pressure and mean blood pressure were significantly normalized and decreased in the BIOCERAMIC group [9].

\section{BIOCERAMIC treatment for relaxation by monitor of autonomous nervous system on heart rate variation (HRV)}

Since stress is often associated with an increase in sympathetic cardiac control and a decrease in parasympathetic control, as a consequence of rapid heart rate and rhythm variability. Another study of human trial of BIOCERAMIC treatment was designed by using wrist monitor of autonomous nervous system to study heart rate variability (HRV) test. The average widening standard deviation of normal-to-normal RR intervals (SDNN) values in pulse waveforms increased with statistical significant. $(p<0.05)$. This finding implies that BIOCERAMIC treatment has the effect of relaxation and controlled by the parasympathetic nervous system and vagus nerve $[10,15]$.

\section{Clinical assessment of BR to alleviate drug withdrawal symptoms of stimulant addiction or overdose of hypnotic drugs}

The clinical application of BR includes patients with overdose or abuse of drugs with history of suicide attempts. It was including a case of 48-year-old female who suffered a major and severe head injury which caused subsequent intracranial hemorrhage leading to a comatose state for several weeks. She thereafter became despondent, suffered depression and came to rely on illegal purchases of amphetamine ( 0.7 to 1.5 grams per day) to ameliorate her depression, fatigue, anxiety and emotional instability. The patient was in poor relationship with family members due to financial affairs. Besides, she also in need of hypnotic drug of flunitrazepam, about $2 \mathrm{mg}$ per night, to help her fall asleep. After consultation in our hospital, treatment of BR was provided to her, with pulsed sound output is set at one cycle of 1 to 12 beats per second (bps), and she was given a treatment session of as 2 times in a week and one cycle lasting $45 \mathrm{~min}$. The subject can choose to repeat specific bps settings based on the intensity of the sensation experienced [8]. About the clinical outcome, social and psychological benefit after BR treatment were include: (1) Regained her spirit, recovered muscular strength and felt like breathing fresh air; (2) Improved with deep sleep pattern; (3)Re-built a good family relationship; (4) dosage of drug after treatment of amphetamine decreased to less than 0.2 grams per day after 5 days and then complete withdraw within 10 days of BR treatment; and (5) flunitrazepam reduced to fundamental maintain dose of $0.5 \mathrm{mg}$ per night.

There was another case of a 62-year-old female, with complain of severe sleep difficulty and severe depression that aggravated by remembering the tragedy of the death of her only son. She was needing a heavy dose of the hypnotics drug of zolpidem at least $70 \mathrm{mg}$ per night. However, she was still easily awakened, even though a minor stimulation by a small sound produced outside the bedroom. We arranged for her to receive BR treatment with the same protocol as case one. According to her statement, the clinical outcome, social and psychological benefit after treatment were included: (1) deep sleep at night; (2) Became sleepy before midnight; (3) Improved of an overwhelming feeling of sadness; (4) Improved of her depression and poor spirit in the morning; and (5) dosage of zolpidem was decreased to $30 \mathrm{mg}$ per night [11].

\section{Clinical assessment of $B R$ with instant beneficial effect on patient with psychopathically related spasmodic torticollis}

Spasmodic torticollis is a kind of debilitating neurological movement disorder with tonic and phasic involuntary movements of the cervical musculature cause intermittent, involuntarily, or sustained deviations and jerky movements of the head and neck. The disorder is always associated with mental problems of somatization, obsessive-compulsive, interpersonal sensitivity, depression, and anxiety [12]. There was a 61-year-old male, who initially began suffering from spasms of his right face and sternocleidomastoid muscle 34 years ago. He complained of twitching and turning of his face to the left side with his chin upwards, and the illness was triggered by psychopathic disease. He had consulted psychiatrists who prescribed medications and provide him with physical therapies for over three decades, but without significant improvement. BR is combined with a tempo of 
sound waves at a frequency of $6 \mathrm{~Hz}$ of about $70-80-\mathrm{dB}$ and PLB with light-emitting diode (LED) irradiation of wavelength spectrum of 300-700 nm with illumination strictly controlled at 500 Lux. After BR\&PLB treatment, there were significant improvements in involuntary movements of patients with spasmodic torticollis measured by motion speed changes and direction complexity (MSCDC). Furthermore, a computerized facial emotional assessment (CFEA) showed that patients expressed greater positive emotions after treatment, with results show a very significant decrease in the percentage of sadness after treatment that represents a negative emotion. There is also found very significant increase in the percentage of surprised which generally represents a positive emotion. Finally, we also performed quantitative assessments on voice analysis of speech signal features (SSF). There were studies of patients with depression had found acoustic features, could be useful as biomarkers for evaluation of symptom severity and treatment efficacy for depression. In our study, it was indicated a significant decrease of fundamental frequency of the patient's SSF after the treatments of BR\&PLB $[12,16]$.

\section{Study the effect of $B R$ on perception, state of consciousness and the related mental activity.}

BR was applied on 155 healthy Taiwanese adults participates who received a questionnaire assessment of consciousness change or subjective response before and after treatments by using BR system. Further observations of selected cases of follow-up BR experiment. Precise descriptions and statistical analysis were performed on the results. It was found negative group at $26.7 \%$ of the participates without recognizable change of the treatment. Amongst the positive group of participates (73.3\%), were reported with specific perceptions, which include: (a) improvement in their sleep quality; (b) subjective sensation through certain parts of the skin; (c) deep swirling and light/color visualizations; and, (d) more intriguing cases involved candidates who had inexplicable phenomena with audio-visual experiences, such as rotating 'mandala'. By using 'The Chi Square Goodness of fit' test, there is highly statistically significant difference between the negative and positive groups $\left(P<0.001^{* *}\right)$. This study attempted to hypothesize that the BR effect may correlate with the Jungian style of psychotherapy and the concept of 'instrumentalization' on mental health care [13].

\section{Clinical effect of BR on psychological related sleep disturbance with findings on questionnaire, EEG and fMRI}

There were 30 female and 15 male participants with complained of insomnia were recruited. They were requested to finish a questionnaire of 'sleep pattern' and 'quality of life', which was modified from the questionnaire of 'Insomnia Severity Index' and 'Pittsburgh Sleep Quality Index'. The modified version can help easy understanding for Taiwanese participants and assessment separately on psychological and physical causes of sleep disturbances. As a result, during the first 3 days of BR treatment, sleep quality was improved in all 36 patients especially for psychological reasons of sleep disturbance. Electroencephalograph (EEG) recordings were also analyzed before, during and after BR treatment. It was found $91.7 \%(33 / 36)$ treatment associated with an elevation in the beta spectrum of the EEG (at 15 to $27 \mathrm{~Hz}$ ) in BR group, compared with $0 \%(0 / 36)$ in the control group. Functional MRI (fMRI) was also used for demonstration of the BR effect for the participants. The result of fMRI found corresponding cerebral and cerebellar areas of activation and deactivation. BR can improve sleep disorder due to psychological causes, with a transient alter brain wave activity and functional activation on specific locations of brain. By comparing the result of the candidates received $3 \mathrm{~T}$ fMRI without and with BR, it showed an fMRI signal increase in areas, include left temporal pole, left parahippocampal gyrus ,left rolandic operculum, left inferior frontal gyrus, left precentral gyrus, left hippocampus, left middle frontal gyrus, left insula, left thalamus, left cerebellum and left gyrus insula. According to our literature reviews, we found these locations activated by BR are related to depression (generalized, unipolar and bipiolar subtypes), sleep deprivation, panic disorder and anxiety disorder [14].

\section{CONCLUSION}

This article summarized our previous studies of using BIOCERAMIC material, BR and PLB on beneficial effects and clinical applications on mental and psychiatric disorders. They were include: (1) alleviate stress condition; (2) relaxation by monitor of autonomous nervous system on HRV; (3) alleviate drug withdrawal symptoms of stimulant addiction or overdose of hypnotic drugs; (4) instant beneficial effect on patient with psychopathically related spasmodic torticollis; (5) effect on perception \& state of consciousness the related mental activity; and (6) beneficial effect on psychological related sleep disturbance with findings on questionnaire, EEG and fMRI.

\section{ACKNOWLEDGMENTS}

This study was supported by a grant (MOHW109-HSO-M211-000001) from Ministry of Health and Welfare, Taiwan.

\section{REFERENCES}

1. Kessler RC, Soukup J, Davis RB, Foster DF, Wilkey SA, et.al. (2001). The use of complementary and alternative therapies to treat anxiety and depression in the United States The American Journal of Psychiatry 158:289-94.

2. Wu P, Fuller C, Liu X, Lee HC, Fan B, et.al. (2007) Use of complementary and alternative medicine among women with depression: results of a national survey. Psychiatric Services 58:349-56. 
3. Beaubrun G, Gray GE (2000) A review of herbal medicines for psychiatric disorders. Psychiatric Services. 51:1130-4.

4. Brown CH, Wohlheiter K, Dixon LB (2005) Use of practitionerbased alternative therapies by psychiatric outpatients. Psychiatric Services. $56: 1460-1$.

5. Spence DW, Kayumov L, Chen A, Lowe A, Jain U, et.al. (2004) Acupuncture increases nocturnal melatonin secretion and reduces insomnia and anxiety: a preliminary report. The Journal of Neuropsychiatry Clinical Neuroscience. 16:19-28.

6. Leung TK. (2015) In vitro and in vivo studies of biological effects of BIOCERAMIC (material of emitting high performance far-infrared ray) Irradiation. Chinese Journal of Physiology 58: 147-155

7. Leung TK, ChanWP, Tai CJ, Cho TP, Yang JC, et.al. (2015) Bioceramic Resonance Effect on Meridian Channels: A Pilot Study. Evidence Based of Complementary and Alternative Medicine. 2015; 769546

8. Chang YC, Leung TK. (2020) Establishment of a basic medical science system for Traditional Chinese medicine education: A suggestion based on the experience of BIOCERAMIC technology. Journal of Traditional and Complementary Medicine 10: 95-103.

9. Leung TK, Chen CH, Tsai SY, Hsiao G, Lee CM. (2012). Effects of far infrared rays irradiated from ceramic material (BIOCERAMIC) on psychological stress-conditioned elevated heart rate, blood pressure, and oxidative stress-suppressed cardiac contractility. Chinese Journal of Physiology. 55: 323-330

10. Liau BY, Leung TK, Ou MC, Ho CK, Lin YS, et.al. (2012)Inhibitory effects of far-infrared ray generating belt on primary dysmenorrheal. International Journal of Photoenergy 2012; 238468:1-6
11. Leung TK, Lee CM, Gasbarri M, Chen YC. (2018). Base on concept of traditional Chinese medicine: Experimental studies on efficacy of BIOCERAMIC Resonance to alleviate drug withdrawal symptoms, Journal of Traditional and Complementary Medicine, 8:257-262.

12. Leung TK, Yang TS, Lin RF, Tsai DM, Chou CM. (2020) Evaluate clinical efficacy of bioceramic technology on psychopathically related spasmodic torticollis using motion measurements, computerized analysis of facial emotions and speech signal features. Biomedical Engineering: Applications, Basis and Communications 32: 2050021 (13 pages)

13. Leung TK. (2020) Bioceramic resonance induced extrasensory perception or altered state of consciousness: A pilot study of Taiwan, Chulalongkorn Medical Journal 64: 291-298.

14. Zhang L. Chan P, Liu ZM, Tseng YL, Chen CW, et.al. (2017) A technology developed from concept of acupuncture and meridian system, the clinical effect of BIOCERAMIC resonance on psychological related sleep disturbance with findings on questionnaire, EEG and fMRI, Journal of Traditional Complementary Medicine 8:289-295.

15. Goldberger JJ, Challapalli S, Tung R, Parker MA, Kadish AH. (2001) Relationship of heart rate variability to parasympathetic effect. Circulation. 103:1977-83.

16. Mundt JC, Snyder PJ, Michael S, Chappie K, Geralts DS.(2007) Voice acoustic measures of depression severity and treatment response collected via interactive voice response (IVR) technology, Journal of Neurolinguistics 20:50-64. 